\title{
Application of electronic nose to effectiveness monitoring of air contaminated with toluene vapors biofiltration process
}

\author{
Bartosz Szulczyński ${ }^{1,}$, Piotr Rybarczyk ${ }^{1}$, and Jacek Gębicki ${ }^{1}$ \\ ${ }^{1}$ Gdańsk University of Technology, Department of Chemical and Process Engineering, Faculty \\ of Chemistry, 11/12 Gabriela Narutowicza Street, 80-233 Gdańsk, Poland
}

\begin{abstract}
The research presents the application of electronic nose (combined with MLR model) to on-line effectiveness monitoring of biofiltration of air contaminated with hydrophobic, odorous compound (toluene vapors). The research was conducted using two-section biotrickling filter inhabited by Candida environmental isolates. Gas chromatography was used as the comparative technique to obtain reliable quantification of toluene concentration in the samples. After about 200 hours of the process, a removal efficiency of $49 \%$ was obtained.
\end{abstract}

\section{Introduction}

The constant economic and industrial development beyond the unquestionable economic benefits also brings threats, especially to the environment. Every human activity is a source of emissions of various compounds, including volatile organic compounds, which enter the atmosphere. Many of them can cause odour nuisances due to their unpleasant smell $[1,2]$.

One of the examples of a compound characterized by negative hedonic tone is toluene belonging to the group of aromatic hydrocarbons. Toluene is one of the most common compounds polluting atmospheric air. It is used in the pharmaceutical and organic industry, as a raw material in the production of dyes, detergents and perfumes. Toluene is also a component of high-octane aviation fuels. It has been proven that it is toxic to humans, has an adverse effect on the respiratory, nervous or immune systems. It can also cause eye and skin irritation and it is also harmful for aquatic organisms. Due to the relatively low odour threshold, even its non-significant concentrations in the air can cause odour nuisance. The basic physicochemical properties of toluene are presented in Table 1.

Table 1. Toluene properties.

\begin{tabular}{|c|c|}
\hline Property & Value \\
\hline Chemical group & Aromatic hydrocarbons \\
\hline Density (at $20^{\circ} \mathrm{C}$ ) & $862.3 \mathrm{~kg} \cdot \mathrm{m}^{-3}$ (liquid) \\
\hline
\end{tabular}

\footnotetext{
${ }^{*}$ Corresponding author: bartosz.szulczynski@pg.edu.pl
} 


\begin{tabular}{|c|c|}
\hline Property & Value \\
\hline Hydrophobicity & Moderate \\
\hline Smell type & Intensive, pleasant \\
\hline Odour threshold & $0.021-2.8 \mathrm{ppm}$ \\
\hline
\end{tabular}

\subsection{Biofiltration}

The issue of counteracting unpleasant odors consists not only in removing odorous contaminations, but also in controlling emission sources and methods for measuring and evaluating emitted odorous gases. Constantly growing public awareness and technical innovations lead to the continuous development of deodorization technology [3]. Deodorization of gases can be done in three ways:

- odours removal,

- conversion of odorants into odourless compound,

- doping, aimed at changing the character of the fragrance or reducing its intensity and hedonic tone.

There are three groups of gas deodorization methods: physical, chemical and biological. Physical and chemical methods of removing odorants are widely used, because the equipment used in such technologies is often small, processes are carried out relatively quickly, the design and operation of this type of equipment has been well-known and can be used in cleaning large waste gas streams containing high concentrations of pollutants [4].

The process of biofiltration consist in the decomposition of gas contaminants by bacteria or other microorganisms inhabiting the porous packing bed of the biofilter. As a result of a gas flow through the packing, the contaminants from the gas phase diffuse to the biofilm surrounded by a liquid (usually aqueous) phase (Figure 1). The liquid phase is either supplied as a trickiling the bed (mobile liquid phase) or it is supported by gas humidification prior to its entering to the biofilter. Pollutants adsorbed on and absorbed by the biofilm undergo biodegradation. The cleaned gas leaves the biofilter $[5,6]$. The most popular types of devices used in biological air treatment include biofilters, bioscrubbers and biotrickling filters (BTF), which enable both the absorption and degradation of gaseous pollutants to take place in one apparatus. BTF is packed with a bed trickled with a liquid, usually enriched with the nutrients for microorganisms. The role of the packing elements is to give a support to the microbial growth and thus enable the biofilm formation.

Biofilters are typically designed for the removal of water-soluble substances. This is accomplished by moisturizing the filter packing elements with water, resulting in formation of hydrophilic biofilm. The efficiency and the rate of removal of hydrophilic compounds (e.gh. toluene) is governed by the rate of their biodegradation by microorganisms inhabiting the biofilm. It is contrary when compounds having greater affinity to the organic than aqueous phase are considered. Biofiltration of hydrophobic compounds proceeds with a much lower yield than hydrophilic compounds and the treatment effectiveness depends on the rate of mass transport of the component from the gas phase to the biofilm. The improvement of biofiltration of hydrophobic compounds is a challenge in biofilter design and it is currently the main research topic in this area [7-9].

The most often used methods for monitoring of the biofiltration efficiency are gas chromatography techniques. However, they are mainly used in laboratory research. Nowadays, there is noticeable a significant increase in the interest in using electronic noses for quantitative and qualitative analysis in environmental monitoring $[10,11]$. Due to the low cost and short time of a single analysis, they have become an alternative to gas 
chromatography. Electronic noses are devices that are supposed to imitate the human sense of smell and are used in many areas of human activity [11]. The use of electronic noses in quantitative analysis determines the need to use calibration models. The task of the model is to link the e-nose sensor signals with the concentration or quality parameter of substance in the analyzed sample [12]. The most commonly used models are: Multiple Linear Regression (MLR), Principal Components Analysis and Regression (PCA and PCR) and Partial Least Squares Regression (PLSR). These methods found successful application for monitoring of odorous compounds concentration changes in biofiltration process [13-16].

The research presents the application of electronic nose (combined with MLR model) to effectiveness monitoring of biofiltration of air contaminated with hydrophobic, odorous compound (toluene vapors). The research was conducted using biotrickling filter inhabited by Candida environmental isolates. Gas chromatography was used as the comparative technique to obtain reliable quantification of toluene concentration in the samples.

\section{Materials and methods}

\subsection{Biotrickling filter set-up}

Investigations were carried out in a two-section BTF system as shown in Figure 1.

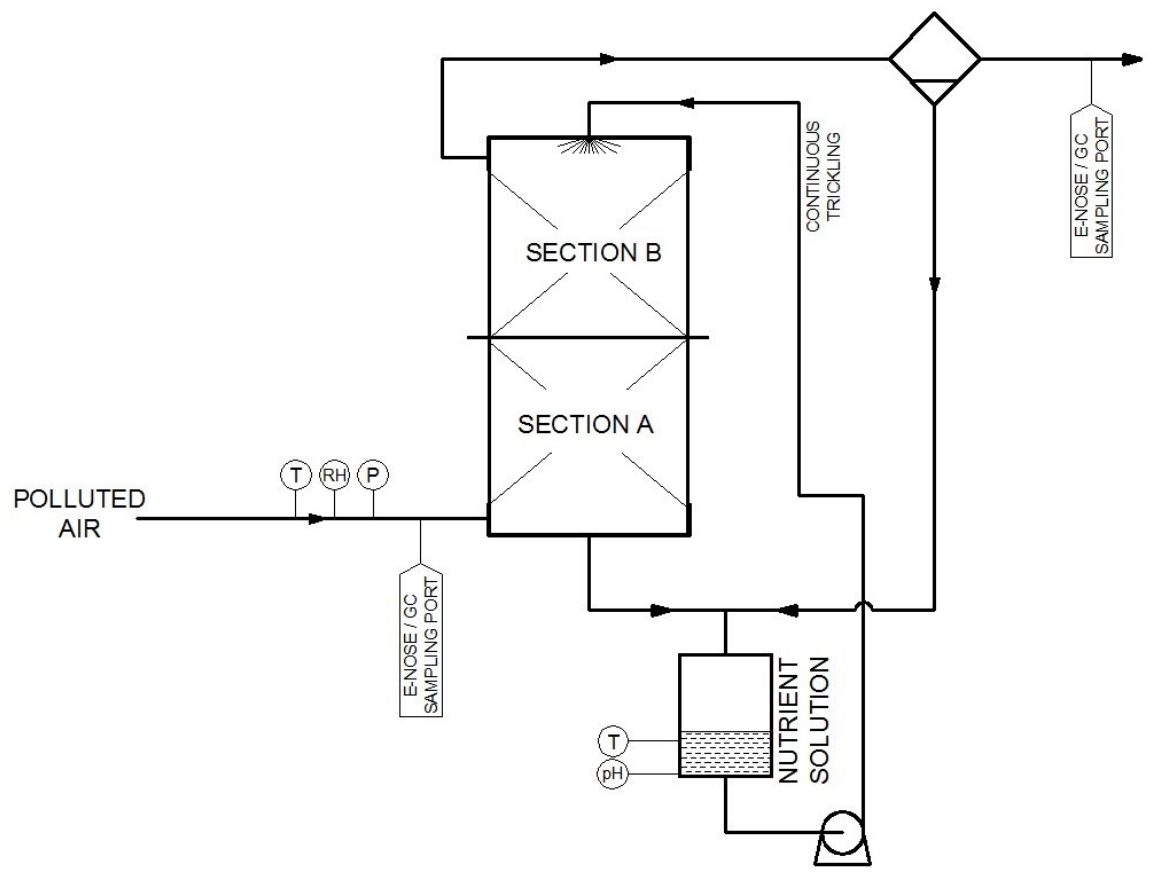

Fig. 1. Biotrickling filter system using during the research.

Basic characteristics of a BTF and a packing elements is given in Table 2.

Table 2. Selected parameters of the biofiltration process.

\begin{tabular}{|c|c|}
\hline Parameter & Value \\
\hline$C_{\text {inlet }}$ (toluene) & $4 \mu 1 \cdot \mathrm{dm}^{-3}$ \\
\hline
\end{tabular}




\begin{tabular}{|c|c|}
\hline Parameter & Value \\
\hline Gas flow rate, $Q_{g}$ & $800 \mathrm{ml} \cdot \mathrm{min}^{-1}$ \\
\hline Biofilter volume, $V$ & $2.8 \mathrm{dm}$ \\
\hline Empty Bed Retention Time $(E B R T)$ & $210 \mathrm{~s}$ \\
\hline Trickling liquid flow rate, $Q_{L}$ & $0.001 \mathrm{dm}^{3} \cdot \mathrm{s}^{-1}$ \\
\hline Trickling frequency & 1 min. every 15. minutes \\
\hline Filter packing elements & Raschig rings $(6 \mathrm{x} 1.5 \mathrm{~mm})$ \\
\hline Packing porosity & $0.512 \pm 0.023$ \\
\hline Packing specific surface area & $1456 \pm 108 \mathrm{~m}^{2} \cdot \mathrm{m}^{-3}$ \\
\hline
\end{tabular}

The BTF was filled ceramic Rashig rings inhabited by selected environmental isolates of Candida species, taken from the collection of environmental Department of Molecular Biotechnology and Microbiology, Chemical Faculty, Gdansk University of Technology. The packing of a BTF i.e. ceramic Rashig rings were inoculated by immersing in $5 \mathrm{dm}^{3}$ of a nutrient solution with Candida species for 4 days. Then, the packing was placed in respective sections of BTF and a nutrient solution was recirculated for additional 7 days prior the startup of the process. A biofilter inhabited by microorganisms is shown in Fig. 2.

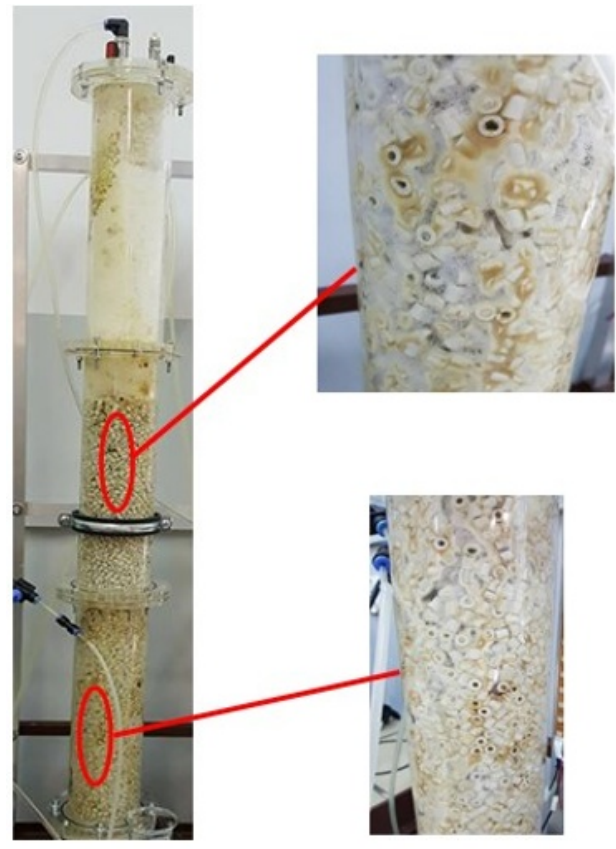

Fig. 2. A biotrickling filter inhabited by Candida microorganisms. 


\subsection{Gas samples analysis}

\subsubsection{Gas chromatography}

Analysis of gas samples were performed using GC-FID system. Gas chromatography method parameters were: DB-WAX column (30 m x 0,53 mm ID x $1 \mu \mathrm{m}$; carrier gas - Nitrogen 1.3 $\mathrm{ml} \cdot \mathrm{min}^{-1}$; oven program: $40^{\circ} \mathrm{C}(5 \mathrm{~min}) / 20^{\circ} \mathrm{C} \cdot \mathrm{min}^{-1} / 200^{\circ} \mathrm{C}(3 \mathrm{~min})$, inject volume: $1 \mu \mathrm{l}$. The external standard method was used as the calibration method (Fig. 3.).

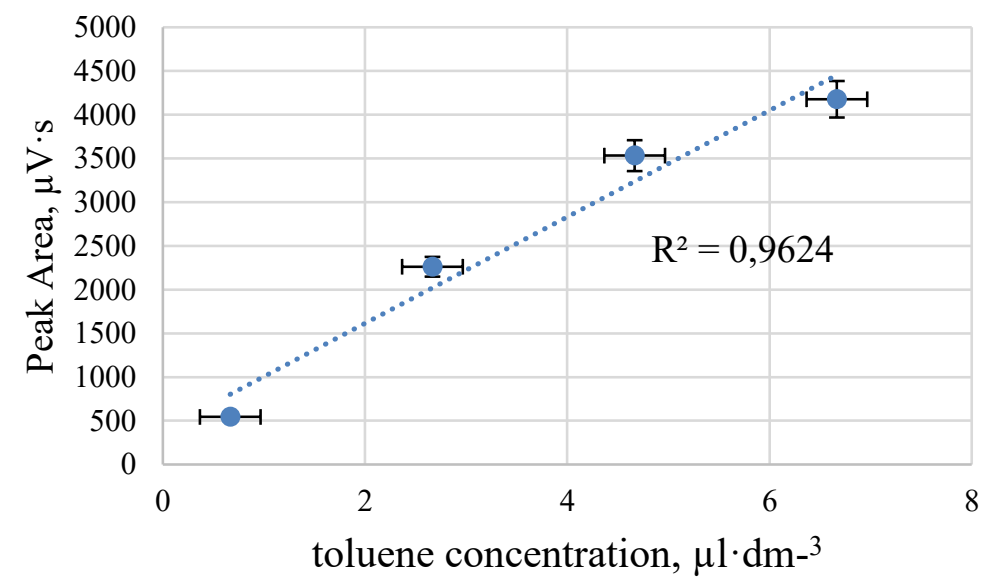

Fig.3. Gas chromatography calibration plot.

\subsubsection{Electronic nose}

For continuous monitoring of the BTF performance the prototype of electronic nose was used. It was equipped with eight chemical sensors (Table 3.).

Table 3. Models of chemical sensors used to build an electronic nose prototype.

\begin{tabular}{|c|c|c|}
\hline Number & Model & Target gas \\
\hline $\mathrm{S}_{1}$ & MiniPID & VOCs \\
\hline $\mathrm{S}_{2}$ & FECS44-100 & Ammonia \\
\hline $\mathrm{S}_{3}$ & FECS50-100 & hydrogen sulphide \\
\hline $\mathrm{S}_{4}$ & TGS2600 & air contaminants \\
\hline $\mathrm{S}_{5}$ & TGS2602 & VOCs and odorous gases \\
\hline $\mathrm{S}_{6}$ & TGS2603 & $\begin{array}{c}\text { air contaminants (triethylamine, } \\
\text { mercaptanes, etc.) }\end{array}$ \\
\hline $\mathrm{S}_{7}$ & TGS823 & \begin{tabular}{c} 
organic solvent vaporous \\
\hline $\mathrm{S}_{8}$
\end{tabular} \\
\hline
\end{tabular}


The recorded sensor signals were saved on the computer using the Simex SIAi- 8 analogto-digital converter. Analyzed sample was sucked by the pump and flowed through the enose system to the measurement chamber at a constant flow rate of $300 \mathrm{~cm}^{3} \cdot \mathrm{min}^{-1}$. The electronic nose operated in stop-flow mode: the sample flow time through the chamber was 60 seconds, while the stop time was 30 seconds. Toluene concentrations were determined using the model based on the Multiple Linear Regression (MLR). Development of the MLR models were described in [17]. Developed model:

$$
\log c=-13.54+9.44 \cdot \log S_{1}-2.25 \cdot \log S_{4}+3.11 \cdot \log S_{7}
$$

was characterized by determination coefficient $\left(\mathrm{R}^{2}\right)$ equal to 0.92 .

\subsection{Calculations}

The effectiveness of the biofiltration process was assessed based on the values of removal efficiency (RE):

$$
R E=1-\frac{C_{\text {out }}}{C_{\text {in }}}
$$

where: $C_{i n}, C_{o u t}-$ concentration of toluene at the inlet and outlet of the BTF, respectively.

\section{Results and discussion}

Fig. 4. shows the dependence of biofiltration effectiveness as a function of process time (determined by electronic nose combined with MLR model (1) and gas chromatography).

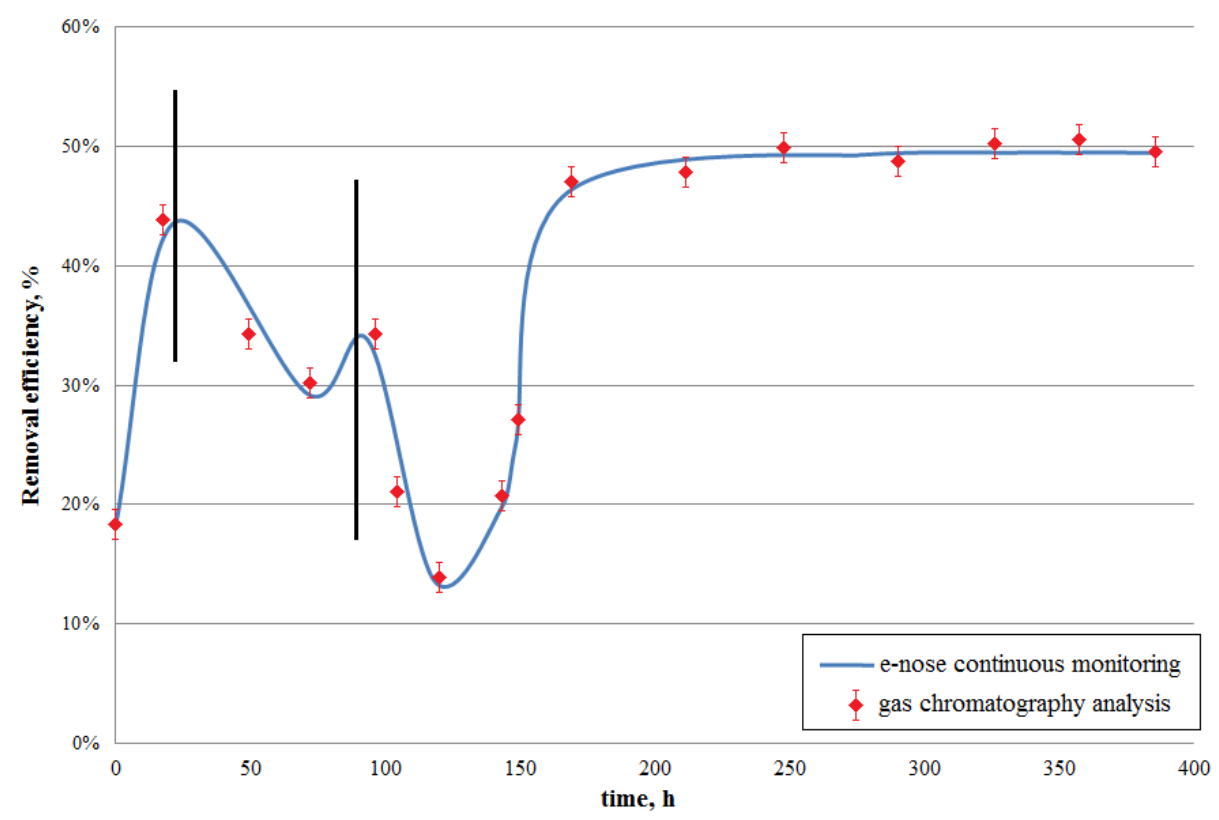

Fig.4. Dependence of biofiltration effectiveness as a function of process time.

Three stages of the process can be distinguished on the plot. In the first stage, the socalled complete medium, that is, rich in nutrients that are necessary for fungi growth. The 
second stage occurred after changing the trickling liquid into a poorer one, first the process shows a decrease in the efficiency of purification, then after the acclimation of microorganisms, the efficiency of biofiltration increases. In the third stage, the nutrient solution that already contained only mineral salts was used. This treatment was aimed at achieving maximum efficiency of biofiltration, because the only carbon source needed in the metabolic processes of Candida fungi remained toluene.

After about 200 hours of the process, a removal efficiency of $49 \%$ was obtained. If no modification of the aqueous phase is used for the surface active compound, this process efficiency is satisfactory and coincides with the literature data [18].

The data obtained during the experiment show very good agreement between the results obtained using electronic nose combined with MLR model and the chromatographic analysis. Small deviations may be caused by relatively high water vapor content in the samples, which may disturb the measurements using the MOS sensors. In addition, there are no possible interferences from other volatile organic compounds.

\section{Conclusions}

Based on the presented studies it was observed that the proposed biofilter type (biotrickling filter) with Rashig rings packed bed and environmental isolates of Candida fungi successfully purifies the air stream from toluene vapors. The $49 \%$ of removal efficiency was obtained, which in such conditions of conducting the process is the desired result. In order to obtain higher values, it would be necessary to modify the aqueous phase with a surfactant that would allow greater availability of toluene for the microorganisms living in the aqueous biofilm.

The application of the electronic nose prototype combined with MLR model allowed to monitor the process in on-line mode. Thanks to the use of gas chromatography, it justifies that the use of an electronic nose is justified for biofiltration effectiveness monitoring. Due to the low cost of the prototype and short time and low cost of a single analysis the use of electronic noses seems to be purposeful for such applications. The possibility of obtaining results in the on-line mode also in the future gives the possibility of using them as measuring elements in automation systems for biofiltration process management.

The investigations were financially supported by the Grant No. UMO-2015/19/B/ST4/02722 from the National Science Centre, Poland.

\section{References}

1. A. Grzelka, I. Sówka, U. Miller, Inż. Ekolog. 2, 56-64 (2018)

2. P. Lewkowska, B. Cieślik, T. Dymerski, P. Konieczka, J. Namieśnik, Environ. Res. 151, 573-586 (2016)

3. P. Henshaw, J. Nicell, A. Sikdar, Atmos. Environ. 40, 1016 (2006)

4. J.M. Estrada, N.J.R.B. Kraakman, R. Muñoz, R. Lebrero, Environ. Sci. Technol. 45, $1100(2011)$

5. D. McNevin, J. Barford, Biochem. Eng. J. 5, 231-242 (2000)

6. K. Chmiel, A.B. Jastrzębski, M. Palica, Przem. Chem. 84, 442-445 (2005)

7. M. Schiavon, M. Ragazzi, E.C. Rada, V. Torretta, Crit. Rev. Biotechnol. 36, 1143-1155 (2016)

8. M. Ferdowsi, A. Avalos Ramirez, J.P. Jones, M. Heitz, Int. Biodeterior. Biodegrad. 119, 336-348 (2017) 
9. Y. Cheng, H. He, C. Yang, G. Zeng, X. Li, H. Chen, G. Yu, Biotechnol. Adv. 34, 10911102 (2016)

10. L. Capelli, S. Sironi, R. Del Rosso, Sensors 14, 19979-20007 (2014)

11. B. Szulczyński, T. Wasilewski, W. Wojnowski, T. Majchrzak, T. Dymerski, J. Namieśnik, J. Gębicki, Sensors 17, 2671 (2017)

12. A.D. Wilson, M. Baietto, Sensors 9, 5099-5148 (2009)

13. B. Szulczyński, K. Armiński, J. Namieśnik, J. Gębicki, Sensors 18, 519 (2018)

14. R. Lopez, I.O. Cabeza, I. Giraldez, M.J. Diaz, Bioresour. Technol. 102, 7984-7993 (2011)

15. B. Szulczyński, P. Rybarczyk, J. Gębicki, Monatsh. Chem. 149, 1693-1699 (2018)

16. I.O. Cabeza, R. Lopez, I. Giraldez, R.M. Stuetz, M.J. Diaz, Chem. Eng. J. 233, 149-158 (2013)

17. B. Szulczyński, J. Gębicki, J. Namieśnik, Chem. Pap. 72, 527-532 (2018)

18. J. Octavio Saucedo-Lucero, R. Marcos, M. Salvador, S. Arriaga, R. Muñoz, G. Quijano, Chemosphere 117, 774-780 (2014) 